EXTENDED REPORT

\title{
In vitro and in vivo killing of ocular Demodex by tea tree oil
}

\author{
Y-Y Gao, M A Di Pascuale, W Li, A Baradaran-Rafii, A Elizondo, C-L Kuo, V K Raju, S C G Tseng
}

Br J Ophthalmol 2005;89:1468-1473. doi: 10.1136/bjo.2005.072363

\begin{abstract}
See end of article for authors' affiliations

.....................

Correspondence to: Scheffer C G Tseng, MD, $\mathrm{PhD}$, Ocular Surface Center, 7000 SW 97 Avenue, Suite 213, Miami, FL 33173, USA; stseng@ ocularsurface.com

Accepted for publication 6 June 2005
\end{abstract}

\begin{abstract}
Aims: To compare the in vitro killing effect of different agents on Demodex and to report the in vivo killing effect of tea tree oil (TTO) on ocular Demodex.

Methods: Survival time of Demodex was measured under the microscope. Sampling and counting of Demodex was performed by a modified method.

Results: Demodex folliculorum survived for more than 150 minutes in $10 \%$ povidone-iodine, $75 \%$ alcohol, $50 \%$ baby shampoo, and $4 \%$ pilocarpine. However, the survival time was significantly shortened to within 15 minutes in 100\% alcohol, 100\% TTO, 100\% caraway oil, or 100\% dill weed oil. TO's in vitro killing effect was dose dependent. Lid scrub with $50 \%$ TO , but not with $50 \%$ baby shampoo, can further stimulate Demodex to move out to the skin. The Demodex count did not reach zero in any of the seven patients receiving daily lid scrub with baby shampoo for 40-350 days. In contrast, the Demodex count dropped to zero in seven of nine patients receiving TTO scrub in 4 weeks without recurrence.

Conclusions: Demodex is resistant to a wide range of antiseptic solutions. Weekly lid scrub with 50\% TTO and daily lid scrub with tea tree shampoo is effective in eradicating ocular Demodex.
\end{abstract}

$\mathrm{T}$ he organism Demodex folliculorum is found in the eyelash follicle and Demodex brevis burrows deep in sebaceous and meibomian glands. ${ }^{1}$ Although their pathogenic role remains unsettled, efforts have been made to eradicate ocular Demodex in patients presenting with blepharitis. Junk et al $^{2}$ reduced the Demodex count in half after 1 month of lid scrub with baby shampoo combined with topical $2 \%$ metronidazole gel. In a case report, Fulk and Clifford ${ }^{3}$ used topical 1\% mercury oxide ointment to bring the Demodex count from 10 mites in eight lashes to one in four lashes in 3 weeks. Subsequently, they used topical $4 \%$ pilocarpine gel to decrease the Demodex count from 2.4 per lash to 0.9 per lash in 2 weeks. ${ }^{4}$ Judged from the fact that the aforementioned treatments do not eradicate ocular Demodex in one life cycle - that is, 14-18 days, ${ }^{5}$ we questioned their capability of killing Demodex in vivo.

We thus surveyed a number of potential agents and discovered that tea tree oil (TTO) is a promising acaricide. TTO, a natural essential oil steam distilled from the leaf of Melaleuca alternifolia, has long been used as an aboriginal traditional medicine in Australia for wounds and cutaneous infection. It has antibacterial, ${ }^{6-9}$ antifungal, ${ }^{9-13}$ antiinflammatory, ${ }^{14}{ }^{15}$ and acaricidal effects. ${ }^{16}$ Shampoo containing TTO has been used to kill lice as well as nits, ${ }^{17}$ and $2.5 \%$ TTO gel is well tolerated and non-toxic for treating dental plaques and chronic gingivitis. ${ }^{18}$ Here, we further showed how weekly lid scrub with $50 \%$ and daily lid scrub with TT shampoo could eradicate ocular Demodex in 1 month in seven of nine patients.

\section{MATERIALS AND METHODS \\ Materials}

Ten per cent povidone iodine, $75 \%$ and $100 \%$ alcohol, mineral oil, 10\% SDS, and TTO were from Sigma (Milwaukee, WI, USA); $4 \%$ pilocarpine solution was from Bausch \& Lomb (Tampa, FL, USA), caraway oil and dill weed oil were from Essential Oil Company (Portland, Oregon, USA). TT shampoo was from Kato Sales, Inc (Altamonte Springs, FL, USA). TTO was diluted with mineral oil into different concentrations.

\section{In vitro killing}

Because there is a high prevalence of Demodex in patients with cylindrical dandruff $(C D),{ }^{19}$ lashes with CD were chosen for the in vitro killing study. Under the microscope, if the epilated lash contained live Demodex, different solutions were added. The movement of Demodex body and legs was continuously observed for 150 minutes. The survival time (ST) was defined as the time when the solution was added to the time when the movement ceased (fig 1). The average ST was compared among different solutions. The value $150 \mathrm{~min}-$ utes was used to denote as no effect, and after that time, we also noted that the Demodex body gradually deformed and shrunk (data not shown). Because Demodex at an earlier stage of life was more vulnerable, we only tested adult Demodex that had four pairs of well developed legs and a stumpy body. Because Demodex buried in CD might preclude the killing effect, we only tested Demodex organisms in which more than half of the body was exposed to the test solution.

\section{Patients}

This study followed the tenets of Helsinki Declaration. Patients with CD at the lashes or who complained of ocular irritation without identifiable causes were recruited. All patients had received routine complete eye examination and external photography, and were found to have ocular Demodex (see below). They were not using topical or systemic anti-inflammatory and antibacterial medications. Seven patients had practised lid scrub with diluted baby shampoo at least once daily.

\section{Lash sampling and Demodex counting}

The method of lash sampling and Demodex counting is detailed in a recent report. ${ }^{19}$

\section{Lid scrub with TTO}

In the office, after we administered a drop of $0.5 \%$ oxybuprocaine (proparacaine), a cotton tip wetted in 50\% TTO was used to scrub the lash roots from one end to the other as one stroke. A total of six strokes were applied to each lid. A dry cotton tip was then used to remove excess TTO from the lid margin. Because TTO scrub not only loosened CD but also stimulated Demodex to move out to the skin (see Results), a dry cotton tip was used to remove CD 5 minutes later. After

Abbreviations: $C D$, cylindrical dandruff; $S T$, survival time; $T T O$, tea tree oil 

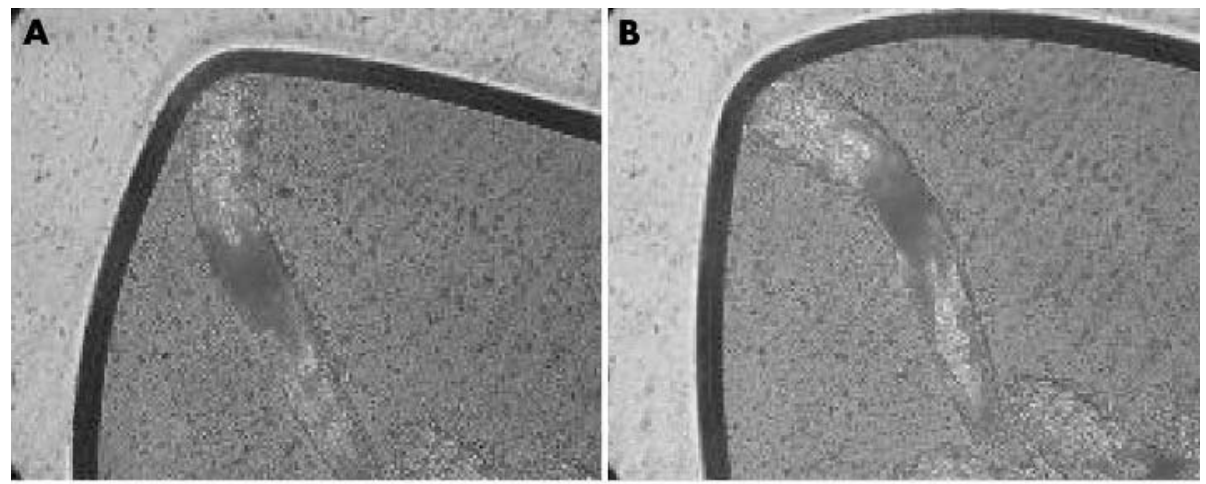

Figure 1 In vitro live and dead status of Demodex. The same Demodex was photographed before $(A, C$, and $E)$ and 5 minutes later ( $B, D$, and $F)$. The Demodex was judged to be "live" by vivid movement of the body $(A, B)$ or legs $(C, D)$. In contrast, the Demodex was judged to be "dead" by the lack of movement $(E, F)$. Furthermore, all eight legs of the dead mite were stretched apart $(E, F)$ distinctively different from the live one $(C, D)$.
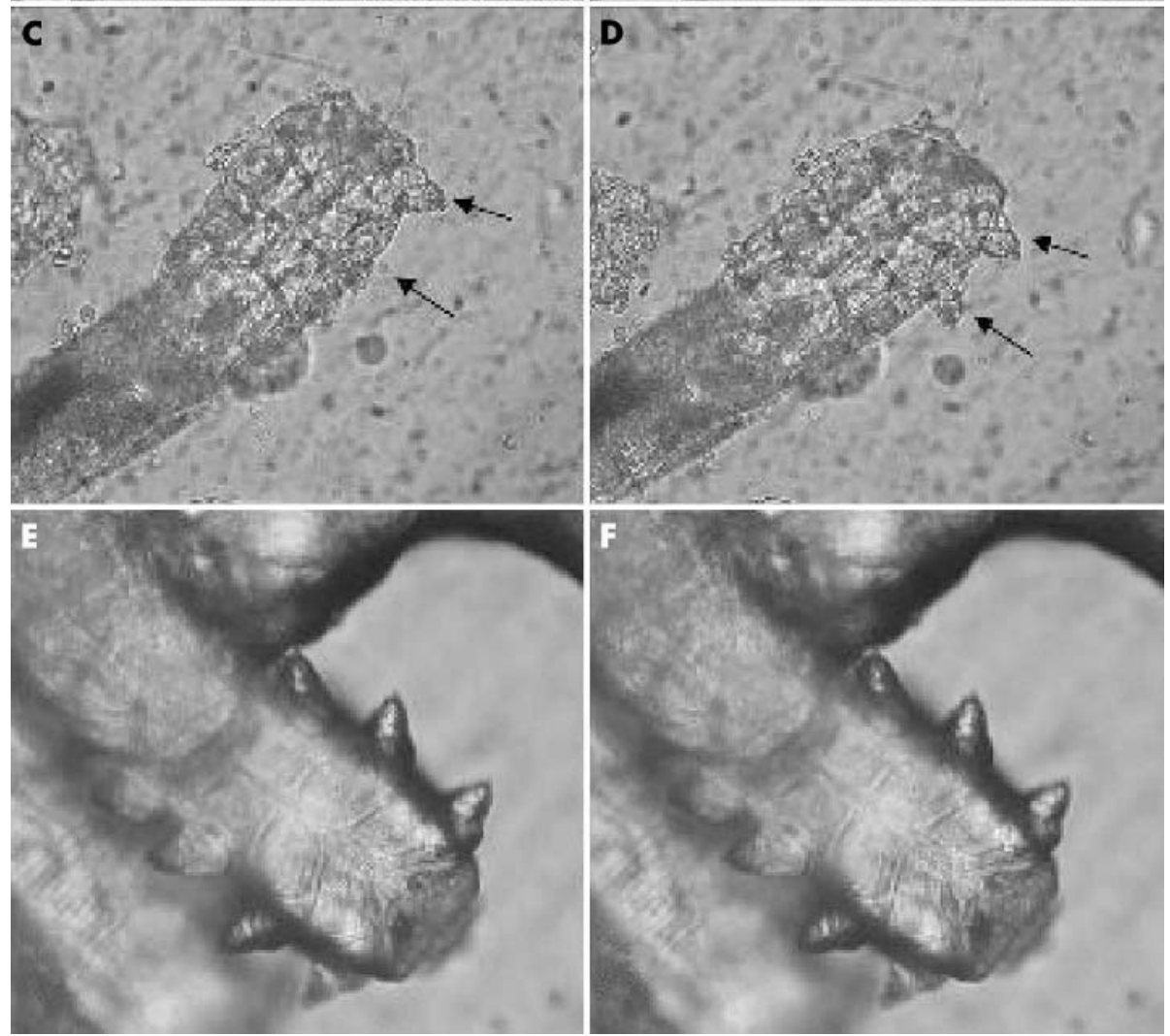

another 5 minutes, the second lid scrub with TTO was applied in the same manner. Following 10 minutes of rest, the third TTO lid scrub was applied. If there was any irritation, the eye was rinsed with non-preserved saline. This office scrub was repeated weekly until the Demodex count reached 0 for two consecutive visits.

\section{Home lid scrub and other instructions}

At home, the patient was asked to mix $0.5 \mathrm{ml}$ of TT shampoo with tap water in both middle fingers. With eyes closed, the lids were massaged with a medium pressure for 3-5 minutes. The skin was then rinsed with clean water and dried with a towel. Such home lid scrub was practised twice daily for 1 month and then once daily thereafter. In addition, the patient was also instructed to discard used facial make-up, and wash the hair, the face, nostrils, the external ear and the neck with TT shampoo daily. The bedding and pillow cases were washed with hot water and dried in a heated dryer immediately after the first office scrub, and once a week thereafter. If the spouse also complained of similar eye irritation, the same home lid scrub was also practised.

\section{Statistical analysis}

The data were reported as means (SD). and analysed by MicroSoft Excel (MicroSoft, Redmont WA, USA). The data between groups were evaluated by two tailed $t$ test where $\mathrm{p}<0.05$ was considered statistically significant.

\section{RESULTS}

\section{In vitro killing effect}

We first surveyed the effect of a number of agents listed in table 1 on $D$ folliculorum, and noted that $50 \%$ baby shampoo, $100 \%$ mineral oil, $75 \%$ alcohol, $10 \%$ povidone-iodine, or $4 \%$ pilocarpine could not kill Demodex in 150 minutes. In contrast, 100\% alcohol killed them in 3.9 (SD 1.2) minutes. Because Demodex is frequently trapped in the CD, which consists of cellular debris made of lipids and keratins, ${ }^{1}$ we also examined whether a pretreatment with such a detergent as $10 \%$ SDS or $50 \%$ baby shampoo might promote the killing effect of other agents. The results showed that none of these pretreatments for 30 minutes could facilitate the killing by subsequent application of either $10 \%$ povidone-iodine or $75 \%$ alcohol. In contrast, $100 \%$ of TTO, caraway oil or dill weed oil 
Table 1 Survival time of Demodex folliculorum in different solutions

\begin{tabular}{|c|c|c|c|c|c|c|c|c|}
\hline Solution & $50 \%$ BS & $10 \% \mathrm{PI}$ & $75 \%$ Alc & MO & 4\%Pilo & Mix 1 & Mix 2 & Mix 3 \\
\hline ST (minutes) & 150 & 150 & 150 & 150 & 150 & 150 & 150 & 150 \\
\hline DN & 9 & 4 & 8 & 5 & 3 & 6 & 5 & 6 \\
\hline Solution & $100 \%$ Alc & 10\% пто & $25 \%$ пा० & $50 \%$ поО & $100 \%$ тाО & $100 \%$ CWO & $100 \%$ DWO & \\
\hline ST (minutes) & $3.9(1.2)$ & 150 & $34.7(4.3)$ & $14.8(9.5)$ & $3.7(0.8)$ & $4.4(2.3)$ & $14.0(8.3)$ & \\
\hline DN & 7 & 5 & 5 & 11 & 21 & 16 & 5 & \\
\hline
\end{tabular}

ST, survival time; DN, number of Demodex tested; BS, baby shampoo; $M O$, mineral oil; Pl, povidone-iodine; Alc, alcohol; Mix 1, $50 \%$ BS in water for 30 minutes followed by $10 \%$ PI; Mix 2, 10\% SDS in water for 30 minutes followed by $10 \% \mathrm{Pl}$; Mix 3, 10\% PI for 30 minutes followed by $75 \%$ Alc; $\mathrm{TO}$, tea tree oil; CWO, caraway oil; DWO, dill weed oil; Pilo, pilocarpine.

killed these mites in $3.7(0.8), 4.4(2.3)$, and $14.0(8.3)$ minutes, respectively. Furthermore, the killing effect of TTO was dose dependent-that is, being 14.8 (9.5), 34.7 (4.3), and 150 minutes for $50 \%, 25 \%$, and $10 \%$ of $\mathrm{TTO}$, respectively $(\mathrm{p}<0.01) ; 50 \%$ TTO and $10 \%$ TTO also exerted a similar killing of $D$ brevis (not shown).

In vivo killing by lid scrub with diluted baby shampoo In seven patients who had been practising daily home lid scrub with diluted baby shampoo for more than 1 month, we found out that the Demodex counts did not reached zero (fig 2). Cases 3, 4, and 5 maintained a constant number of Demodex after 40-350 days of lid hygiene. Cases 1 and 7 actually showed an increase of Demodex after 110-350 days. Only cases 2 and 6 showed a decrease of Demodex after 64 and 125 days of lid hygiene, respectively, but never reached zero.

\section{In vivo killing by lid scrub with TTO}

We recently confirmed that lashes with CD are pathognomonic for ocular Demodex. ${ }^{19}$ It is thus important to get rid of $\mathrm{CD}$, which harbours Demodex. In a patient presenting with symmetrical signs of CD in both eyelids, lid scrub with $50 \%$ TTO in the right eye and with $50 \%$ baby shampoo in the left eye for 5 minutes equally cleaned off CD (fig 3A and B). However, Demodex was found on the skin surface adjacent to the lashes (fig 3C, arrows), and a Demodex tail was seen protruding from the lash root (fig 3C, arrowheads) in the TTO treated eye, but not in the baby shampoo treated eye (fig 3D). Furthermore, the skin in the eye receiving TTO was redder

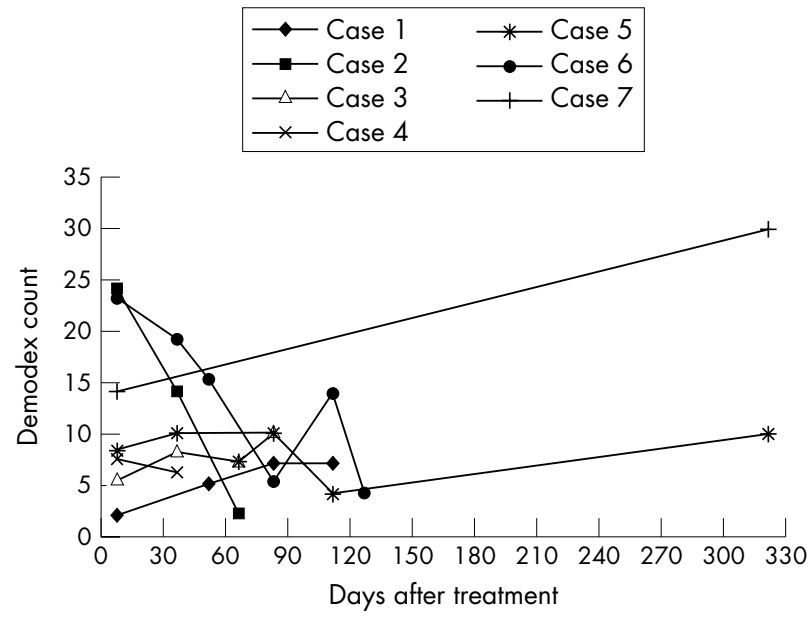

Figure 2 Demodex counts in patients receiving lid scrub by diluted baby shampoo. In these seven patients, their serial Demodex counts did not drop to zero despite daily lid scrub with diluted baby shampoo when practised for more than 1 month. Cases 3, 4, and 5 maintained a constant Demodex count around 7-10 after 40-350 days of lid scrub. Cases 1 and 7 had an increase of Demodex count after 110 and 350 days of lid scrub. Cases 2 and 6 showed a decrease of Demodex count after 64 and 125 days of lid hygiene, respectively, but did not reach zero. than that receiving baby shampoo. These findings suggested that lid scrub with TTO did not merely mechanically remove CD from the lash root, but might attract mites to move out to the skin.

To examine this possibility, we epilated lashes with diffuse CD (fig 4A) and found that $D$ folliculorum clustered around the lash follicle (fig 4D). After office lid scrub with 50\% TTO, the lashes were free of CD (fig 4B). Interestingly, tails were protruding from the lash root (fig $4 \mathrm{~B}$, arrow). When these lashes were epilated, $D$ folliculorum was found on the lash trunk close to the skin surface-that is, away from the lash follicle (fig 4E). When such lash was rotated by a forceps before epilation, we noted several mites migrating along the lash trunk (fig $4 \mathrm{~F}$ and $4 \mathrm{G}$ ). If no home lid scrub was subsequently practised, CD returned in 1 week (fig 4C). If the lashes were scrubbed with baby shampoo, simple mechanical rotation did not stimulate Demodex to move out (not shown). These results indicated that lid scrub with $50 \%$ TTO, but not baby shampoo, indeed stimulated Demodex to migrate from $\mathrm{CD}$ embedded inside the skin.

Because lid scrub with 50\% TTO did not kill mites buried deep in the skin, and because if no lid scrub was carried out at home, CD quickly returned in l week, we advocated that weekly office lid scrub with $50 \%$ TTO be followed by daily home lid scrub with TT shampoo in nine patients (fig 5). The results showed that the Demodex count dropped to zero in 3 weeks in five patients and in 4 weeks in another two patients without any recurrence 1 month later. These seven patients were younger (59.86 (8.7) year old), and had a mean count of 7.9 (4.1) before treatment. In the remaining two patients, cases 1 and 2, the count did not reach zero, and showed a rebound 35 days and 14 days after treatment, respectively. These cases were 68 and 81 years old, and had a higher count of 26 and 16, respectively, before treatment. The rebound count was less than the previous one, and the interval between rebounds was $2-3$ weeks.

\section{DISCUSSION}

Applying our recently modified sampling method, we detected Demodex in $100 \%(\mathrm{n}=32)$ of patients presenting $\mathrm{CD}$ at eye lashes, and in $22 \%(\mathrm{n}=23)$ of those with clean lashes. ${ }^{19}$ In a randomly selected general population $(n=206)$, the incidence of Demodex on lids and the nose is $4 \%$ in those younger than 19 years old, $30 \%$ in those between 20 and 80 years old, and $47 \%$ in those older than 80 years old. ${ }^{20}$ However, no research has convincingly demonstrated whether a minimal number of mites sampled must be present in order to produce symptoms.

Consistent with our earlier report, ${ }^{19}$ we noted that the Demodex count in all seven patients receiving daily lid hygiene with baby shampoo never reached zero in a period between 40 and 350 days - that is, beyond one life cycle (fig 2). These results, together with those published earlier, ${ }^{24}$ strongly imply that the conventional treatment cannot eradicate Demodex, a notion also supported by the finding that $50 \%$ baby shampoo did not kill Demodex in vitro (table 1). It should also be noted that $75 \%$ alcohol and $10 \%$ povidone-iodine, 

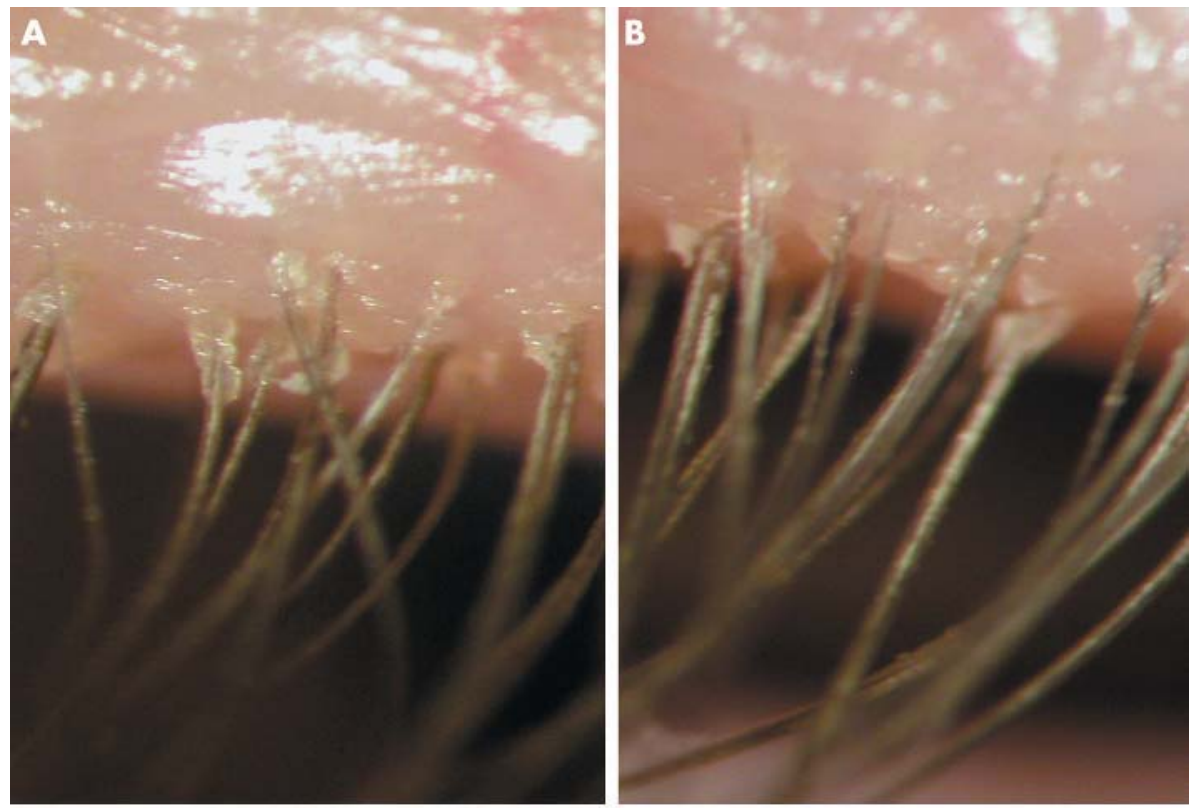

Figure 3 Different results of lid scrub by $T O$ and baby shampoo. In a patient presenting with symmetrical signs of $C D$ in both eyelids, lid scrub was performed in the office by $50 \%$ TTO in the right eye (A) and by $50 \%$ baby shampoo in the left eye (B). In either eye, $C D$ was clean after scrub (C, D), however, Demodex could be found on the lid margin adjacent to the lashes ( $C$, arrows), and a Demodex tail could be visualised protruding from the lash root (C, arrowheads) after TTO scrub. These findings were not detected in the eye after baby shampoo scrub although the skin surface was less red (D).
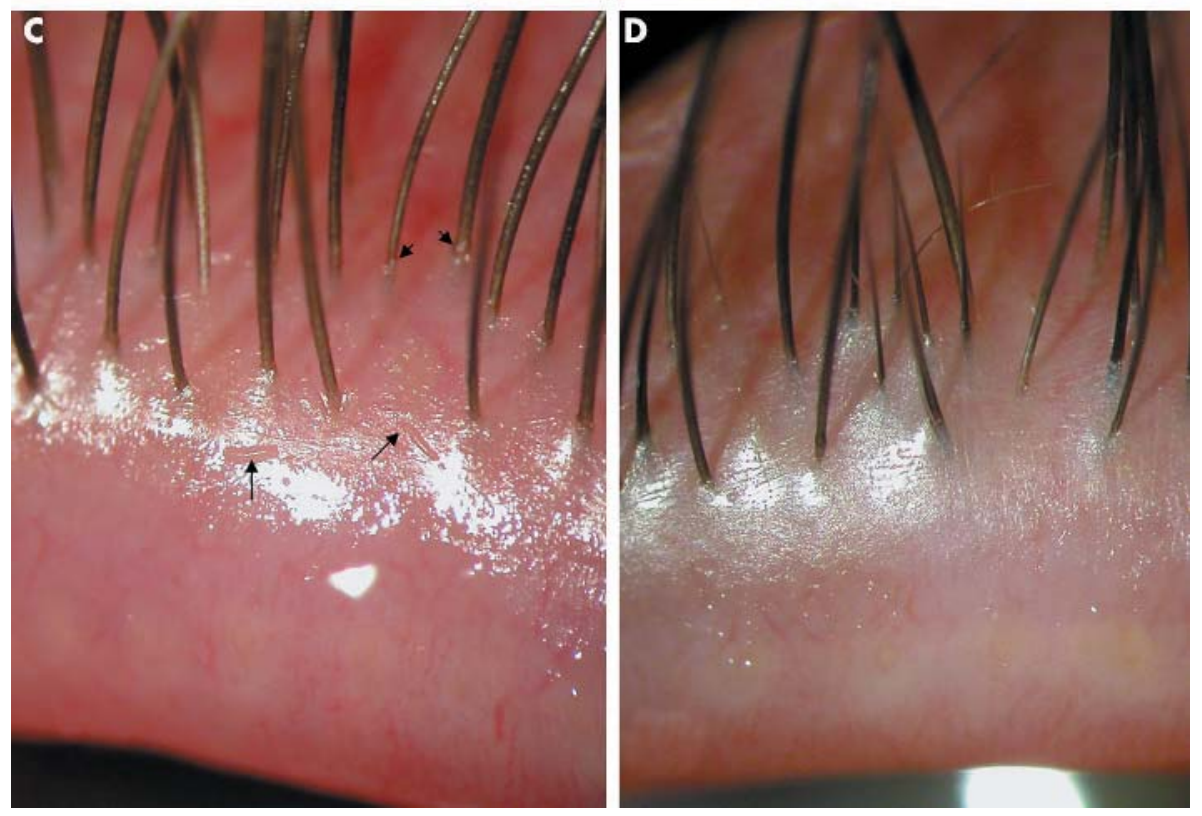

agents known to kill most microbes, could not kill Demodex in vitro (table 1). Because previous incubation with $50 \%$ baby shampoo or $10 \%$ SDS, detergents dissolving oil, before $10 \%$ povidone-iodine still could not kill Demodex (table 1), we suspect that Demodex is resistant to povidone-iodine. Although 4\% pilocarpine gel was used to treat Demodex, ${ }^{4}$ it did not kill Demodex in 150 minutes (table 1).

Norn $^{21}$ tested 45 different agents and found only a few could kill Demodex within several minutes-absolute alcohol, ether, xylol, benzene, Danish (sulfur-containing) ointment, dill weed oil, and caraway oil. We also noted that $100 \%$ alcohol killed Demodex in 3.9 (1.2) minutes. Furthermore, $100 \%$ of dill weed oil and caraway oil-that is, essential oils, also killed Demodex in 14 (8.3) and 4.4 (2.3) minutes, respectively (table 1). Unfortunately, these agents are not amenable for clinical use because of their intrinsic toxicity (irritation) to the eye.

We were encouraged by TTO's killing effect, which resembled that of caraway oil and exhibited a clear dose dependent relation (table 1). Although the killing effect of TTO on lice is thought to be mediated by anti-cholinesterase activity, ${ }^{22}$ it cannot explain its killing effect on nits. TTO might cause contact dermatitis and allergy, ${ }^{23}{ }^{24}$ but the dermal LD50 exceeds $5 \mathrm{~g} / \mathrm{kg}$ in rabbits, and undiluted TTO does not produce phototoxic effects on the skin of hairless mice. ${ }^{25}$ TTO patch test does not cause adverse reactions. ${ }^{26}$ These results encouraged us to use TTO to treat ocular Demodex. TT shampoo is commercially available for treating head lice without an adverse effect.

Although both effectively cleaned CD, TTO, but not baby shampoo, stimulated Demodex to migrate from the CD buried inside the skin (figs 3 and 4). However, if daily lid scrub were not followed, CD returned in l week (fig 4). Because mechanical agitation to the lash following TTO scrub was sufficient to stimulate Demodex to migrate out to the skin, we advocate daily lid scrub following each office lid scrub with TTO. Because neither $50 \%$ baby shampoo nor TT shampoo, of which the concentration of TTO is less than $10 \%$, could kill 

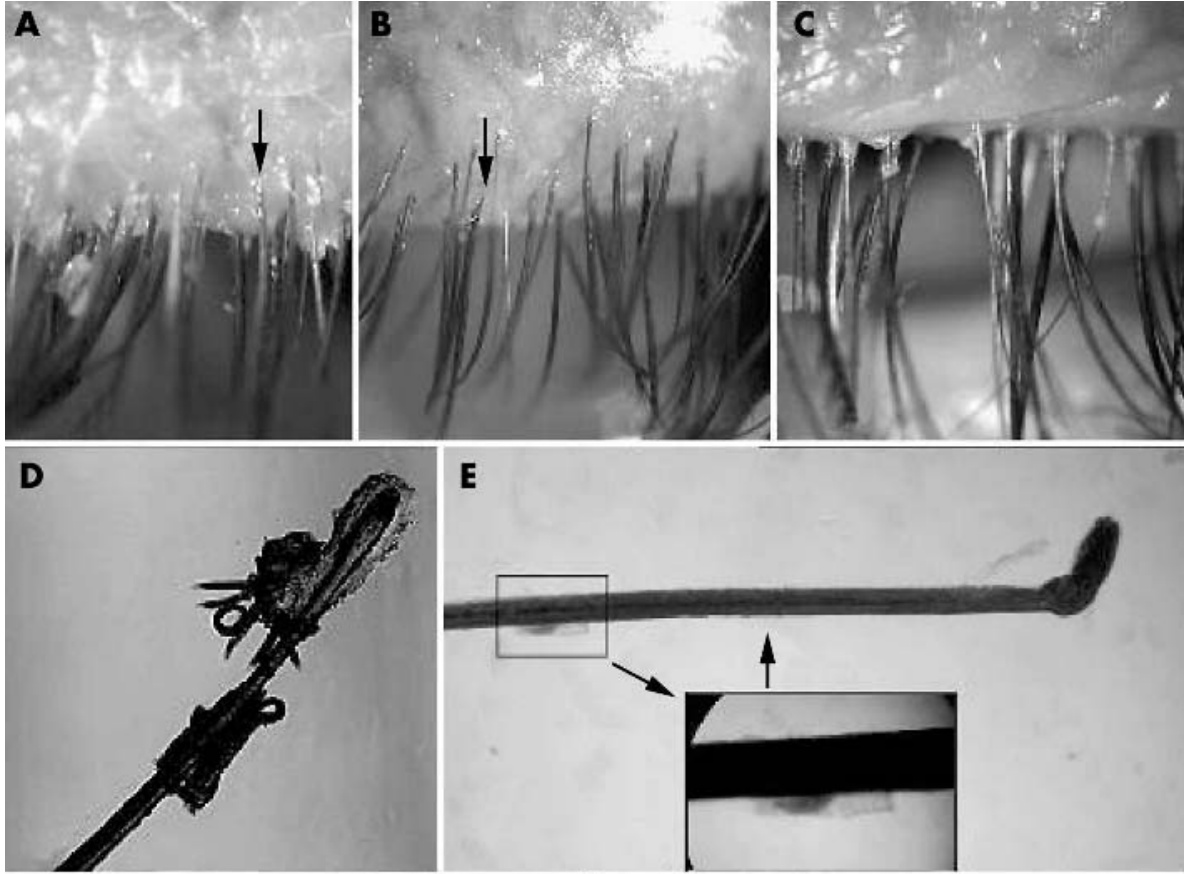

$\mathbf{E}$

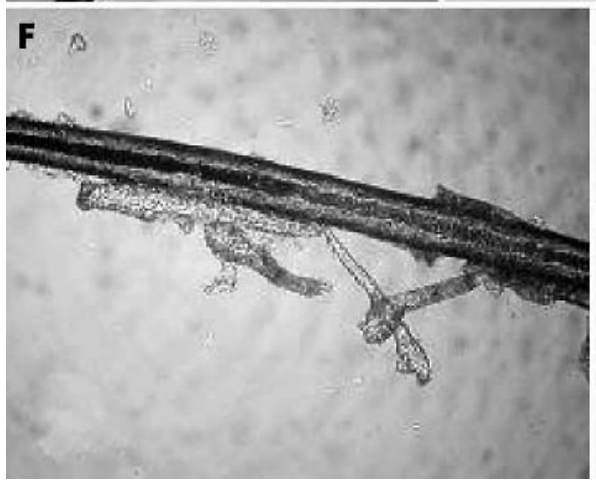

G

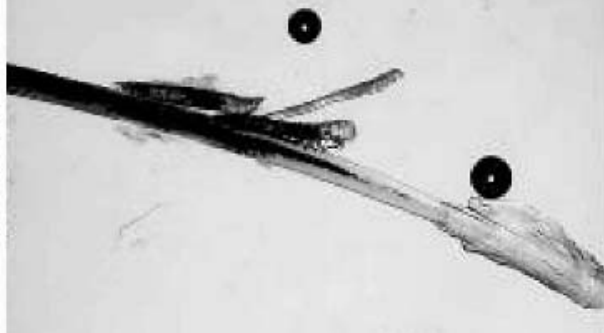

Figure 4 Migration of Demodex by lid scrub with TTO. In this eye with diffuse $C D$ found in the lashes before treatment (A), the lash to be epilated (marked by arrow) showed a fragment of CD attached to the lash and abundant Demodex embedded close to the lash follicle (D). After office lid scrub with $50 \%$ TTO, the lashes became clean, and totally free of CD, but tails of Demodex were protruding from the lash roots $(B$, arrow). Three minutes after lid scrub, free Demodex were found on the trunk close to the skin surface-that is, away from the lash follicle in the epilated lash (E). Rotating these lashes (shown in B) before epilation allowed us to detect a group of Demodex migrating along the lash trunk ( $F$ and $G$ ). If no lid scrub was carried out at home for 1 week, CD returned to the lashes 1 week later $(C)$.

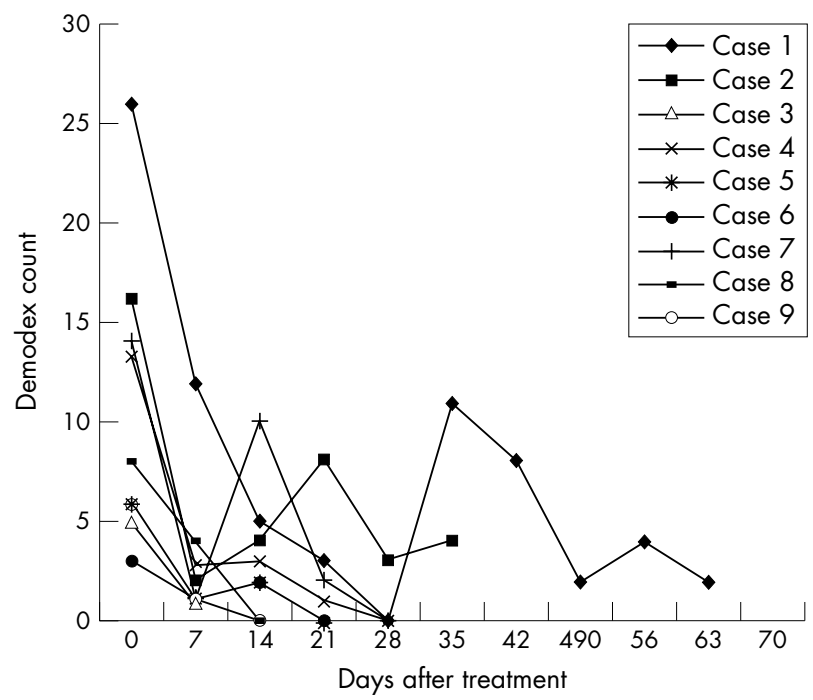

Figure 5 Demodex counts in patients receiving lid scrub with TTO. In these nine patients who received weekly TTO lid scrub plus daily lid scrub with TT shampoo, the Demodex counts dropped sharply in the first week after TTO treatment for all patients. Demodex counts drop to zero in 3 weeks for five patients and in 4 weeks in another two patients, but did not reach zero in two patients at day 35 and day 63 .
Demodex in vitro (table 1), we believe that home lid scrub acts by arousing Demodex to move out and eliminates them before mating.

Using the proposed scrub regimen, the Demodex count was dramatically reduced in 1 week and reached zero in 1 month without recurrence in seven of nine patients (fig 5). The two patients in whom treatment failed were older, started with a higher Demodex count, and rebound in an interval of 23 weeks. Norn, ${ }^{21}$ and our recent studies $^{19}$ have noted that patients with a higher Demodex count tend to be older, suggesting that Demodex infestation, if untreated, tends to be worsened with age because of continuous propagation by mating. We thus speculate that higher Demodex infestation produces more CD inside and outside the lash follicle, and with more resistance to TTO. If this interpretation were correct, we predict that it is easier to treat Demodex at an early stage. Alternatively, the failure might be caused by reinfestation by mites migrating from other places of the body or from the spouse. Because the rebound count was less than the previous one, we speculate that continuous lid scrub might eventually eradicate Demodex.

Lid scrub with 50\% TTO did generate variable degrees of irritation in some patients, but could be minimised if caution is exercised to avoid spilling into the eye. We were encouraged by the finding that patients' symptoms were relieved, ocular surface inflammation was resolved, and the lipid tear film stability improved in these patients (in 
preparation). Collectively, these findings strongly suggest that ocular Demodex infestation might indeed be pathogenic and warrants further controlled studies.

\section{ACKNOWLEDGEMENTS}

The authors thank Javier Moreno, Hua He, Armand Hornia, and Tetsuya Kawakita for their helpful comments on the manuscript.

\section{Authors' affiliations}

Y-Y Gao, M A D Pascuale, W Li, A Baradaran-Rafii, A Elizondo, C-L Kuo, V K Raju, S C G Tseng, Ocular Surface Center and Ocular Surface Research and Education Foundation, Miami, FL, USA

Y-Y Gao, Department of Ophthalmology, the Second Affiliated Hospital, Fujian Medical University, Quanzhou, Fujian, China 362000

Proprietary interest: none

Supported in part by an unrestricted grant from Ocular Surface Research and Education Foundation, Miami, FL, USA.

\section{REFERENCES}

1 English FP, Nutting WB. Demodicosis of ophthalmic concern. Am J Ophthalmol 1981;91:362-72.

2 Junk AK, Lukacs A, Kampik A. Topical administration of metronidazole gel as an effective therapy alternative in chronic Demodex blepharitis - a case report. Klin Monatsbl Augenheilkd 1998;213:48-50.

3 Fulk GW, Clifford C. A case report of demodicosis. J Am Optom Assoc 1990:61:637-9.

4 Fulk GW, Murphy B, Robins MD. Pilocarpine gel for the treatment of demodicosis - a case series. Optom Vis Sci 1996;73:742-5.

5 Spickett SG. Studies on Demodex folliculorum. Simon (1842) 1961;51:181-92.

6 Messager S, Hammer KA, Carson CF, et al. Assessment of the antibacterial activity of tea tree oil using the European EN 1276 and EN 12054 standard suspension tests. J Hosp Infect 2005;59:113-25.

7 Edwards-Jones V, Buck R, Shawcross SG, et al. The effect of essential oils on methicillin-resistant Staphylococcus aureus using a dressing model. Burns 2004;30:772-7.
8 Halcon L, Milkus K. Staphylococcus aureus and wounds: a review of tea tree oil as a promising antimicrobial. Am J Infect Control 2004;32:402-8.

9 Oliva B, Piccirilli E, Ceddia T, et al. Antimycotic activity of Melaleuca alternifolia essential oil and its major components. Lett Appl Microbiol 2003;37: 185-7.

10 Gupta AK, Nicol K, Batra R. Role of antifungal agents in the treatment of seborrheic dermatitis. Am J Clin Dermatol 2004;5:417-22.

11 Hammer KA, Carson CF, Riley TV. Antifungal effects of Melaleuca alternifolia (tea tree) oil and its components on Candida albicans, Candida glabrata and Saccharomyces cerevisiae. J Antimicrob Chemother 2004;53:1081-5.

12 Martin KW, Ernst E. Herbal medicines for treatment of fungal infections: a systematic review of controlled clinical trials. Mycoses 2004;47:87-92.

13 Hammer KA, Carson CF, Riley TV. Antifungal activity of the components of Melaleuca alternifolia (tea tree) oil. J Appl Microbiol 2003;95:853-60.

14 Caldefie-Chezet F, Guerry M, Chalchat JC, et al. Anti-inflammatory effects of Melaleuca alternifolia essential oil on human polymorphonuclear neutrophils and monocytes. Free Radic Res 2004;38:805-11.

15 Carson CF, Riley TV. Safety, efficacy and provenance of tea tree (Melaleuca alternifolia) oil. Contact Dermatitis 2001;45:65-7.

16 Walton SF, McKinnon M, Pizzutto S, et al. Acaricidal activity of Melaleuca alternifolia (tea tree) oil: in vitro sensitivity of sarcoptes scabiei var hominis to terpinen-4-ol. Arch Dermatol 2004;140:563-6.

17 McCage CM, Ward SM, Paling CA, et al. Development of a paw paw herbal shampoo for the removal of head lice. Phytomedicine 2002;9:743-8.

18 Soukoulis S, Hirsch R. The effects of a tea tree oil-containing gel on plaque and chronic gingivitis 1. Aust Dent J 2004:49:78-83.

19 Gao YY, Di Pascuale M, Li W, et al. High prevalence of Demodex in eye lashes with cylindrical dandruff. Invest Ophthalmol Vis Sci 2005:46:3094-8

20 Norn MS. Incidence of Demodex folliculorum on skin of lids and nose. Acto Ophthalmol (Copenh) 1982;60:575-83.

21 Norn MS. Demodex folliculorum. Incidence and possible pathogenic role in the human eyelid. Acta Ophthalmol Suppl 1970;108:7-85

22 Mills C, Cleary BJ, Gilmer JF, et al. Inhibition of acetylcholinesterase by tea tree oil. J Pharm Pharmacol 2004;56:375-9.

23 Simpson EL, Law SV, Storrs FJ. Prevalence of botanical extract allergy in patients with contact dermatitis. Dermatitis 2004;15:67-72.

24 Crawford GH, Sciacca JR, James WD. Tea tree oil: cutaneous effects of the extracted oil of Melaleuca alternifolia. Dermatitis 2004;15:59-66.

25 Ford RA, Letizia C, Api AM. Monographs on fragrance raw materials. Food Chem Toxicol 1988;26:273-415.

26 Brenan JA, Dennerstein GJ, Sfameni SF, et al. Evaluation of patch testing in patients with chronic vulvar symptoms. Australas J Dermatol 1996;37:40-3. 